\title{
Hemipelvectomia no tratamento de condrossarcoma no acetábulo de cão
}

\author{
Hemipelvectomy in the treatment of chondrosarcoma of the acetabulum dog
}

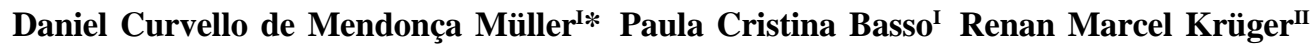 \\ Anne Santos do Amaral ${ }^{\mathrm{III}}$ Cristiano Gomes ${ }^{\mathrm{I}}$ Ney Luis Pippi ${ }^{\mathrm{II}}$
} \begin{abstract}
Hemipelvectomia é a remoção de parte da pelve e
tem sido indicada no tratamento cirúrgico de tumores malignos. RESUMO Este estudo apresenta o caso de um paciente canino, portador de condrossarcoma recidivante, submetido à hemipelvectomia parcial com amputação do membro. O objetivo deste trabalho é ressaltar a importância da cirurgia radical no tratamento de condrossarcoma ósseo, abordando uma alternativa viável e pouco convencional para pacientes acometidos por tumores na pelve, que frente à extensão da lesão podem ser erroneamente diagnosticados como portadores de neoplasias não tratáveis. Após a realização da hemipelvectomia parcial com amputação do membro, o animal obteve retorno satisfatório à deambulação, sem dificuldade de apoio ou alteração de equilíbrio.
\end{abstract}

Palavras-chave: neoplasia, pelve, cirurgia oncológica, cães.

\section{ABSTRACT}

Hemipelvectomy is the removal of part of the pelvis and is indicated for the surgical treatment of malignant tumors. This study presents the case of a canine patient with recurrent chondrosarcoma, submitted to hemipelvectomy with limb amputation. The report aims to highlight the importance of surgery in the treatment of radial bone chondrosarcoma, addressing a viable and unconventional alternative for patients suffering from tumors in the pelvis, which against the extension of the lesion may be misdiagnosed as intractable cancer. After the partial hemipelvectomy with amputation of the limb the animal satisfactory returned to ambulation without difficulty in supporting or alteration in balance.

Key words: neoplasm, pelvis, cancer surgery, dogs.
Os ossos da região pélvica possuem baixa ocorrência de neoplasias malignas. Apesar disso, apresentam particularidades em seu tratamento em razão da complexidade anatômica da região e da dificuldade para sua ressecção com margem adequada (LOPES et al., 1997). O condrossarcoma está entre os três tumores que mais acometem a região pélvica (LOPES et al., 1997). Representa cerca de $10 \%$ do total das neoplasias ósseas diagnosticadas no cão (THOMPSON \& POOL, 2002). É uma neoplasia maligna na qual, células cartilaginosas tumorais produzem quantidades variáveis de matriz fibrilar neoplásica (COUTO, 2006), podendo se desenvolver primariamente em sítios esqueléticos (THOMPSON \& POOL, 2002; ROMANUCCI et al., 2005) ou raramente extraesqueléticos (MILLER et al., 2005; VOORWALD et al., 2008). O tratamento dessa neoplasia é cirúrgico, com remoção do segmento acometido, abrangendo amplas margens sadias (KAWAI et al., 1998). Contudo, não raramente, pacientes que apresentam tumores infiltrados em grandes áreas, principalmente na pelve e nas estruturas adjacentes, não são tratados em razão da complexidade anatômica e da dificuldade de excisão (ROMANO et al., 2004).

A hemipelvectomia é um tratamento cirúrgico adequado para casos de condrossarcoma

\footnotetext{
IPrograma de Pós-graduação em Medicina Veterinária, Universidade Federal de Santa Maria (UFSM), 97105-900, Santa Maria, RS, Brasil. E-mail: cmdaniel@terra.com.br.*Autor para correspondência.

IIPrograma de Residência em Medicida Veterinária, UFSM, Santa Maria, RS, Brasil.

IIIDepartamento de Clínica de Pequenos Animais (DCPA), UFSM, Santa Maria, RS, Brasil.
} 
pélvico, constituindo-se um procedimento no qual é possível remover em monobloco os segmentos ósseos, além de órgãos e estruturas adjacentes que se apresentam macroscopicamente comprometidas (ROMANO et al., 2004).

Este relato tem por objetivos ressaltar a importância da ressecção dentro de princípios oncológicos para lesões que são fundamentalmente de tratamento cirúrgico e abordar aspectos da técnica de hemipelvectomia no tratamento do condrossarcoma acetabular.

Foi atendida uma cadela de cinco anos de idade, sem raça definida, pesando $12 \mathrm{~kg}$ e apresentando aumento de volume com cerca de $25 \mathrm{~cm}$ de diâmetro, na região acetabular do membro pélvico esquerdo. Demonstrava dificuldade em apoiar o membro e de assumir posição para urinar. Essa paciente possuía, em seu histórico, a extração de um nódulo do trocanter maior do fêmur esquerdo há 12 meses, diagnosticado histologicamente como osteocondromatose, e desde então tem sido observado o crescimento de uma massa envolvendo toda a porção proximal do membro, incluindo a face interna da coxa e região inguinal. Realizou-se o exame citológico da massa por meio de punção aspirativa com agulha fina e identificando-se a presença de abundante matriz condroide rósea ao redor de células ovais, isoladas ou em pequenos agrupamentos, apresentando núcleos hipercromáticos com anisocariose acentuada e vacúolos citoplasmáticos discretos, sugestivo de condrossarcoma.

Ao exame radiográfico da pelve nas incidências lateral e ventro-dorsal, evidenciou-se proliferação de tecido radiopaco ao redor do acetábulo com destruição da cabeça e do colo femoral (Figuras 1a e 1b). As imagens do tórax não sugeriam evidências de metástases pulmonares. Foram realizados hemograma e provas bioquímicas para avaliação da função hepática e renal, não se encontrando alterações nesses parâmetros. Com base no histórico do paciente e nos exames realizados, optou-se pela intervenção cirúrgica.

Após medicação pré-anestésica com associação de maleato de acepromazina $\left(0,05 \mathrm{mg} \mathrm{kg}^{-1}\right)$ e sulfato de morfina $\left(0,5 \mathrm{mg} \mathrm{kg}^{-1}\right)$, aplicados por via intramuscular, o animal foi submetido à indução com propofol (4mg kg-1) e manutenção com isofluorano vaporizado em $100 \%$ de $\mathrm{O}_{2}$ durante todo o procedimento.

Com o paciente em decúbito lateral, foram realizadas a antissepsia e colocação dos campos cirúrgicos. Para a exérese da neoplasia com margens de tecidos saudáveis, foi realizada uma incisão elíptica ao redor de todo o membro, excisando-se acima do trocanter maior do fêmur parte da musculatura abdominal ventral e lateral. Realizou-se a osteotomia do ílio em seu ponto mais cranial, utilizando-se serra óssea manual e preservando a articulação sacro-ilíaca e a asa do ílio. Foram seccionados também o púbis e o ísquio (Figuras 1c e 1d), permitindo a remoção do membro em bloco juntamente com a articulação coxofemoral.

Após a remoção do membro e de toda a massa aderida, obteve-se a criação de uma hérnia abdominal, com exposição do omento. Procedeu-se ao fechamento do defeito com tela de polipropileno para impedir a migração das vísceras da cavidade abdominal. Essa tela foi suturada com pontos isolados de fio monofilamentar em toda a periferia da ferida, envolvendo as fáscia musculares e as extremidades ósseas seccionadas. Nas porções ósseas, confeccionou-se um orifício para a passagem da agulha. Isso permitiu aplicar tensão à tela, garantindo a estabilidade da parede. Posteriormente, suturou-se o subcutâneo com pontos isolados utilizando-se fio de ácido poliglicólico em várias camadas. A pele foi suturada com pontos do tipo Wolff, com fio monofilamentar não absorvível. No período pós-operatório, foram prescritos cefalexina (30 $\left.\mathrm{mg} \mathrm{kg}^{-1} \mathrm{BID}\right)$ por 15 dias, cetoprofeno (1,1 $\mathrm{mg} \mathrm{kg}^{-1}$ SID) por quatro dias e cloridrato de tramadol (4mg kg-1 TID) associado à dipirona (25 $\mathrm{mg} \mathrm{kg}^{-1}$ ) por quatro dias. Dez dias após a cirurgia, removeram-se os pontos de pele e foi prescrita alta médica. O exame histopatológico confirmou a suspeita citológica de condrossarcoma. A paciente foi avaliada durante sete meses e nesse período não foram evidenciados sinais de recidiva tumoral.

Os tumores primários da pelve representam cerca de $5 \%$ de todos os tumores ósseos em humanos, mas considerando apenas os condrossarcomas 25\% dos casos estão nessa localização (TERABE et al., 2005). No cão, mais de $60 \%$ dos condrossarcomas têm sido identificados em ossos chatos, como costelas e pelve (POPOVITCH et al., 1994; BAILEY, 2007). Geralmente apresenta evolução clínica lenta, sendo o processo metastático infrequente e tardio (PENNA et al., 1996). No presente relato, o paciente não apresentava sinais evidentes de metástase em tórax e regiões adjacentes.

PENNA et al. (1996) afirmaram que, em seres humanos, os sintomas incluem dor e aumento de volume local. No cão, os condrossarcomas pélvicos produzem claudicação (THOMPSON \& POOL, 2002), que pode variar de mínima até aquela que impede o apoio do membro (BAILEY, 2007). No paciente deste relato, apesar de haver claudicação moderada, a dor à 


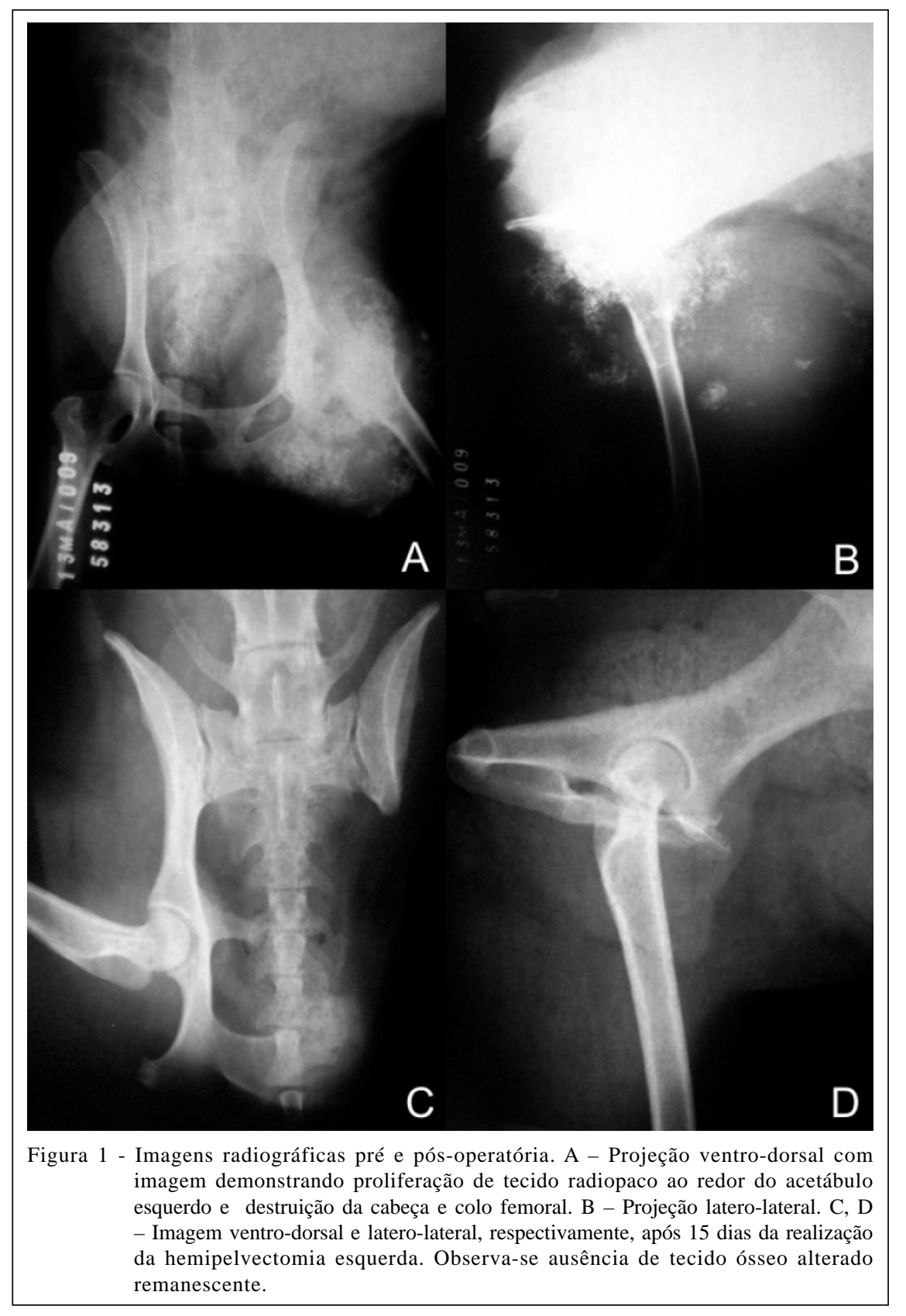

palpação não era evidente na região alterada. O aumento de volume de consistência firme abrangia coxa, região inguinal até a linha média do abdômen e caudal à cicatriz umbilical. Medialmente era possível palpar a massa comprimindo as vísceras abdominais e dificultando a micção do animal. A compressão visceral ocorre ocasionalmente quando a massa pélvica é suficientemente grande (THOMPSON \& POOL, 2002). Segundo PENNA et al. (1996) e THOMPSON \& POOL (2002), o tumor pode ser classificado quanto à localização em central (quando surge a partir do osso) ou periférico (quando surge do periósteo). Condrossarcoma secundário surge a partir da transformação maligna de uma lesão óssea anterior (THOMPSON \& POOL, 2002). Não foi possível determinar a exata origem do tumor em razão da extensa infiltração e lesão das estruturas adjacentes, mas pelo histórico de osteocondromatose o mais provável é que se tratasse de um condrossarcoma secundário. Segundo BAILEY (2007), a osteocondromatose pode sofrer transformação maligna para condrossarcoma e, menos frequentemente, para osteossarcoma. 
Os condrossarcomas apresentam uma variada aparência radiológica, o que torna frequentemente impossível sua distinção dos osteossarcomas (THOMPSON \& POOL, 2002). Radiologicamente a lesão apresenta osteólise e reação periosteal e pode conter pontos de calcificação e ossificação intralesional e espessamento da cortical (KEALY \& McALLISTER, 2005). A radiografia, embora inespecífica, poderá apresentar informações valiosas ao cirurgião, pois, quanto mais radiotransparente o tumor, mais alta a probabilidade de que seja de alto grau de malignidade (GOMES et al., 2006). Um tumor de crescimento lento e de baixo grau causa espessamento reativo do córtex, gerando um aumento de volume endurecido como o apresentado no animal relatado. Por sua vez, uma neoplasia de alto grau, mais agressiva, destrói o córtex e forma uma massa de tecidos moles (GOMES et al., 2006).

Os critérios adotados para o diagnóstico de condrossarcoma em animais é baseado nos critérios estabelecidos para seres humanos (THOMPSON \& POOL, 2002). Segundo PENNA et al. (1996), histologicamente o condrossarcoma se apresenta como um dos mais difíceis ao diagnóstico, pois o critério de diferenciação entre os de baixo grau e o condroma é incerto. Em pacientes veterinários, a diferenciação histológica de tumores mesenquimais envolvendo cartilagens abrange o condrossarcoma, osteossarcoma e outros tipos de sarcomas (THOMPSON \& POOL, 2002).

O tratamento consiste na ressecção cirúrgica com margens amplas, visto que a grande maioria dos casos não responde a outras modalidades de terapia (TERABE et al., 2005). Alguns autores sugerem protocolos quimioterápicos de acordo com o grau de diferenciação e graduação histológica para condrossarcomas pouco diferenciados e condrossarcomas de grau III, porém afirmam que o tratamento é essencialmente cirúrgico (PENNA et al., 1996). A graduação histológica não é feita rotineiramente nos pacientes veterinários, apesar de ser descrita por alguns autores (BRODEY et al., 1974). A excisão cirúrgica radical da lesão é recomendada sempre que possível, e a radioterapia adjuvante pode auxiliar o controle de recidivas locais, apesar de haver pouca informação sobre sua eficácia (BAILEY, 2007). Não existe informação a respeito da eficácia da quimioterapia para o tratamento do condrossarcoma em pacientes veterinários (BAILEY, 2007). Em virtude do tamanho e comprometimento de estruturas adjacentes no caso apresentado, optou-se pela excisão cirúrgica ampla da neoplasia com amputação do membro pélvico associada à remoção total do acetábulo e remoção parcial do ílio, púbis e ísquio. Foi proposto um protocolo quimioterápico adjuvante com associação doxorrubicina e carboplatina, porém em razão da baixa eficácia comprovada, dos efeitos colaterais e do custo o proprietário recusou essa opção.

A hemipelvectomia em pessoas portadoras de condrossarcoma teve excelentes resultados pósoperatórios, com volta à deambulação sem auxílio de muletas em alguns pacientes não amputados (PENNA et al., 1996; ROMANO et al., 2004). Os autores também afirmaram que a adaptação de quadrúpedes amputados já é conhecida no meio veterinário, uma vez que ocorre a triangulação dos membros remanescentes, gerando um equilíbrio adequado. No presente relato, foi possível obter deambulação satisfatória e reestabelecimento adequado da função urinária. A taxa de cirurgias ablativas, em 42 anos de rotina do Hospital A.C. Camargo de São Paulo, com 149 pessoas portadoras de condrossarcoma, foi de $48,7 \%$, em decorrência de excisão insuficiente e do grande volume dos tumores (PENNA et al., 1996). Problema semelhante foi observado no paciente veterinário descrito neste relato, que anteriormente sofreu intervenção cirúrgica paliativa, impossibilitando adoção de medidas conservadoras para o tratamento.

O principal fator prognóstico para recorrência local é a realização do procedimento cirúrgico sem margens adequadas (KAWAI et al., 1998). Com base nessa informação, optou-se pelo procedimento com amplas margens. Outros fatores que devem ser considerados são o grau histológico, a invasão neurovascular e o tratamento inicial incorretamente realizado, que pode elevar os índices de recidivas em até 3,3 vezes (SHETH et al., 1996). Quanto ao tratamento anteriormente realizado nesse paciente, não foi possível determinar a sua abrangência, mas, segundo relato do proprietário, o animal recebeu alta com uma pequena incisão de pele, o que sugere um procedimento mais conservador.

O cão foi avaliado mensalmente durante sete meses e não foi observado macroscopicamente aumento de volume nas regiões próximas ao local da cirurgia que possam sugerir recidiva. Foram realizados estudos radiográficos mensais da região pélvica, não sendo visibilizadas imagens sugestivas de crescimento tumoral. Além disso, o animal apresentou-se clinicamente saudável, desempenhando adequadamente suas funções fisiológicas e locomoção. Esse resultado foi similar ao descrito por outros autores, em que $62 \%$ dos animais com neoplasias pélvicas apresentaram taxa de sobrevida livre de doença de 12 meses após o procedimento cirúrgico (STRAW et al., 1992). Portanto, foi possível concluir que a obtenção de margens 
cirúrgicas livres pode ser uma alternativa eficaz e viável para o tratamento de neoplasias malignas da região pélvica de cães.

\section{REFERÊNCIAS}

BAILEY, D.B. Chondrosarcoma. In: COTÉ, E. Clinical veterinary advisor: dogs and cats. Saint Louis: Mosby Elsevier, 2007. p.202-204.

BRODEY, R.S.et al. Canine skeletal chondrosarcoma: a clinicalpathologic study of 35 cases. Journal of American Veterinary Medical Association, Schaumburg, v.165, p.6878, 1977.

COUTO, C.G. Distúrbios da cavidade nasal. In: NELSON. R.N.; COUTO, C.G. Medicina interna de pequenos animais. Rio de Janeiro: Elsevier, 2006. Cap.15, p.181-182.

GOMES, A.C.N. et al. Condrossarcoma em paciente com osteocondromatose múltipla: relato de caso e revisão da literatura. Radiologia Brasileira, São Paulo, v.39, n.6, p.449-451, 2006. Disponível em: <http://www.scielo.br/scielo.php?pid=S010039842006000600015\&script=sci_arttext $>$. Acesso em: 9 mar. 2010. doi: 10.1590/S0100-39842006000600015.

KAWAI, A. et al. Prognostic factors for patients with sarcomas of the pelvic bones. American Cancer Society, Baltimore, v.82, n.5, p.851-859, 1998.

KEALY, J.K.; McALLISTER, H. Ossos e articulações. In: Radiologia e ultra-sonografia do cão e do gato. 3.ed. Barueri: Manole, 2005. Cap.5, p.339-411.

LOPES, A. et al. Chondrosarcoma secondary to hereditary multiple exostosis treated by extended internal hemiplevectomy. São Paulo Medical Journal. v.115, n.3, p.1440-1443, 1994. Disponível em: <http://www.scielo.br/ scielo.php? s cript = sci_art text \& pid = S 1516 31801997000300007>. Acesso em: 9 mar. 2010. doi: 10.1590/ S1516-31801997000300007.

MILLER, J.W. et al. Primary splenic mesenchymal chondrosarcom a in a dog. Canadian Veterinary Journal, Ottawa, v.46, p.163-165, 2005. Disponível em: <http://
www.ncbi.nlm.nih.gov/pmc/articles/PMC1082865/pdf/ cvj46pg163.pdf>. Acesso em: 9 mar. 2010.

PENNA, V. et al. Condrossarcoma: experiência do Hospital A.C. Camargo nos últimos 42 anos. Revista Brasileira de Ortopedia, São Paulo, v.31, n.11, p.888-890, 1996. Disponível em: <http://www.rbo.org.br/pdf/1996_nov_88.pdf>. Acesso em: 9 mar. 2010

POPOVITCH, C.A. et al. Chondrosarcoma: a retrospective study of 97 dogs (1987-1990). Journal of American Animal Hospital Association, Lakewood,v. 30, p.81-85, 1994.

ROMANO, M. et al. Hemipelvectomia interna alargada no tratamento de condrossarcoma pélvico localmente avançado: relato de caso. Revista Brasileira de Ortopedia, São Paulo, v.39, n.3, p.132-136, 2004. Disponível em: <http:// www.rbo.org.br/pdf/2004_mar_13.pdf>. Acesso em: 9 mar. 2010.

ROMANUCCI, M. et al. Cutaneous extraskeletal mesenchymal chondrosarcoma in a cat. Veterinary Dermatology, Oxford, v.16, n.2, p.121-124, 2005.

SHETH, D.S. et al. Condrosarcoma of the pelvis. Prognostic factors for 67 patients treated with definitive surgeryh. American Cancer Society, Baltimore, v.78, n.4, p.745750, 1996.

STRAW, R.C. et al. Partial or total hemipelvectomy in the management of sarcomas in nine dogs and two cats. Veterinary Surgery. Philadelphia, v.21, n.3, p.183-188, 1992.

TERABE, F. et al. Condrossarcoma de ramo púbico superior simulando hérnia inguinal. Revista do Colégio Brasileiro de Cirurgia, Rio de Janeiro, v.32, n.6, p.353-344, 2005. Disponível em: <http:// www.scielo.br/scielo.php?script=sci_arttext\&pid=S0100$69912005000600015 \& \operatorname{lng}=p t \& n r m=$ iso\&tlng=pt $>$. Acesso em 9 mar. 2010. doi: 10.1590/S0100-69912005000600015.

THOMPSON, K.G.; POOL, R.R. Tumors of bones. In: MEUTEN, D.J. Tumors in domestic animals. Ames: Iowa State, 2002. Chap.5, p.245-317.

VOORWALD, F.A. et al. Condrossarcoma primário em glândula mamária de cadelas: relato de casos. Veterinária e Zootecnia, Botucatu, v.15, n.3, p.33-34, 2008. 